\title{
Mating structures for genomic selection breeding programs in aquaculture
}

\author{
Anna K. Sonesson ${ }^{1 *}$ (D) and Jørgen Ødegård ${ }^{1,2}$
}

\begin{abstract}
Background: In traditional family-based aquaculture breeding, each sire is mated to two dams in order to separate the sire's genetic effect from other family effects. Factorial mating involves more mates per sire and/or dam and result in more but smaller full- and/or half-sib families. For traits measured on sibs of selection candidates, factorial mating increases intensity of selection between families when selection is on traditional best linear unbiased prediction (BLUP) estimated breeding values (TRAD-EBV). However, selection on genome-wide estimated breeding values (GW-EBV), uses both within- and between-family effects and the advantage of factorial mating is less obvious. Our aim was to compare by computer simulation the impact of various factorial mating strategies for truncation selection on $T R A D-E B V$ versus GW-EBV on rates of inbreeding, accuracy of selection and genetic gain for two traits, i.e. one measured on selection candidates (CAND-TRAIT) and one on their sibs (SIB-TRAIT).

Results: Sire:dam mating ratios of 1:1,2:2 or 10:10 were tested with 100, 200 or 1000 families produced from a constant number of parents (100 sires and 100 dams), and a mating ratio of 1:2 with 200 families produced from 100 sires and 200 dams. With GW-EBV, changing the mating ratio from 1:1 to 10:10 had a limited effect on genetic gain (less than $5 \%$ ) for both CAND-TRAIT and SIB-TRAIT, whereas with TRAD-EBV, selection intensity increased for SIB-TRAIT and genetic gain increased by 41 and $77 \%$ for schemes with 3000 and 12,000 selection candidates, respectively. For both $G W-E B V$ and TRAD-EBV, rates of inbreeding decreased by up to $30 \%$ when the mating ratio was changed from 1:1 to 10:10 for schemes with 3000 to 12,000 selection candidates. Similar results were found for alternative heritabilities of SIB-TRAIT and total number of tested sibs.

Conclusions: Changing the sire:dam mating ratio from 1:1 to 10:10 increased genetic gain substantially with TRAD$E B V$, mainly through increased selection intensity for the SIB-TRAIT, whereas with GW-EBV, it had a limited effect on genetic gain for both traits. Rates of inbreeding decreased for both selection methods.
\end{abstract}

\section{Background}

In selective breeding, the procedures used to select parents and mate the selected parents are important for achieving genetic progress. In traditional family-based aquaculture breeding programs, the number of tanks (full-sib families) is a cost limitation. Many of the traditional family-based aquaculture breeding programs apply a sire:dam mating ratio of $1: 2$, i.e. each sire is mated to two dams in order to separate the sire's genetic effect from other family effects, tank effects (since families are kept in separate tanks until physical tagging), maternal

\footnotetext{
*Correspondence: Anna.Sonesson@nofima.no

${ }^{1}$ Nofima AS, P.O. Box 5010, 1432 Ås, Norway

Full list of author information is available at the end of the article
}

effects (e.g. egg quality), and dominance genetic effects. For aquaculture species, control of reproduction is possible by artificial stripping of eggs and milt, and natural mating can occur and result in mating ratios that differ from 1:1, i.e. factorial mating. Woolliams [1] showed that factorial mating designs increase genetic gain without increasing inbreeding compared to a 1:1 sire:dam mating ratio for schemes using truncation selection on traditional best linear unbiased prediction (BLUP) breeding values with a constant number of parents.

For aquaculture populations, traits that require invasive phenotyping methods (carcass and disease resistance traits) are necessarily measured on sibs of the candidates. Usually, there are 15 to 100 test individuals used per family to estimate the breeding values of the untested 
candidates. However, with factorial mating designs in which the number of mates per sire and/or dam is larger, a potential reduction in the number of full-sibs is compensated by larger numbers of paternal and maternal half-sibs.

The standard criteria used in selective breeding are BLUP estimated breeding values (EBV) based on phenotype and pedigree data [2]. For traits that cannot be measured on selection candidates, but that are instead measured on sibs of candidates, traditional BLUP assigns identical breeding value predictions to all nonphenotyped members of a family. However, if genomewide estimated breeding values [3] are used, different breeding values are obtained for each individual within a family and thus both within- and between-family genetic variation are used for selection. As a consequence, less emphasis is given to the between-family component [4]. Therefore, with traditional BLUP estimated breeding values, factorial mating is expected to increase the intensity of selection between families on traits that are measured on sibs of the candidates (since more families are available for the same number of parents). However, for genome-wide estimated breeding values, even for traits that are measured on sibs of the candidates, selection is done both within and between families and the advantage of factorial mating is less obvious.

Our aim was to compare by computer simulation the impact of various factorial mating strategies for truncation selection on traditional BLUP EBV versus genomewide EBV on rates of inbreeding, accuracy of selection and genetic gain for two traits, i.e. one measured on selection candidates and one on their sibs. We applied random mating of sires and dams among the selected sires and dams within defined mating ratios.

\section{Methods}

\section{Simulation of the historical population}

A historical population with an effective population size $\left(N_{e}\right)$ of 1000 was simulated for 4000 generations according to Fisher-Wright's population model [5, 6]. Five hundred males and 500 females were randomly selected and mated using sampling with replacement. The last generation of these 4000 generations was used as the first generation (Generation 0 ) of the selection population of the breeding scheme.

\section{Simulation of the breeding scheme between Generationo and Generation 10}

Between Generation0 and Generation10, the selection population was simulated as follows. Individuals were split into one group of selection candidates of 3000, 6000 or 12,000 (Ncand) and one group of 3000 or 1000 test individuals (Ntest). Two traits were considered: CANDTRAIT, a trait measured on the selection candidates and SIB-TRAIT, a trait that requires an invasive method and was measured on test individuals, which were assumed to be sacrificed during the recording process. Parents were selected from among the selection candidates based on their respective traditional BLUP (TRAD-EBV) or genome-wide $(G W-E B V)$ estimated breeding values. For the $G W-E B V$-based schemes, the Ncand group constituted the reference population for CAND-TRAIT and the Ntest group constituted the reference population for the SIB-TRAIT (see Table 1 for the respective tested family sizes).

One hundred sires and 100 dams were selected with varying sire:dam mating ratios of $1: 1,2: 2$ or $10: 10$ that produced 100, 200 or 1000 full-sib families, respectively. With a mating ratio of 1:2, 100 sires and 200 dams were selected that produced 200 families. The Ncand and Ntest were split equally over the full-sib families (see Table 1 for family size). Throughout this paper, we define factorial mating as designs that apply sire:dam mating ratios that differ from 1:1, i.e. where one sire is mated to several dams and/or one dam is mated to several sires.

\section{Genome}

Briefly, the genome structure of all individuals was diploid with ten chromosomes, each with a size of $100 \mathrm{cM}$ (see [7] for more details). The infinite sites mutation model [8] was used to create new bi-allelic single nucleotide polymorphisms (SNPs), using a mutation rate of $10^{-8}$ per nucleotide [9] and assuming 1,000,000 nucleotides per cM. SNPs followed Mendel's law of inheritance, and the Haldane mapping function [10] was used to simulate recombination events. For each trait, 50 SNPs per chromosome were sampled randomly to be quantitative trait loci (QTL) (sampling without replacement from SNPs with a minor allele frequency (MAF) $>0.05$, resulting in an average MAF of 0.17 ). From the remaining SNPs, the 1000 SNPs with the highest MAF were chosen as genetic markers. The latter resulted in a total of 10,000 SNPs spread over $1000 \mathrm{cM}$. Reduced numbers of SNPs were obtained by selecting every 2nd SNP, resulting in 5000 SNPs.

Table 1 Family sizes used in the simulated schemes (numbers of candidates or sibs)

\begin{tabular}{lccc}
\hline Sire:dam mating ratio & $\mathbf{3 0 0 0}$ & $\mathbf{6 0 0 0}$ & $\mathbf{1 2 , 0 0 0}$ \\
\hline 1:1, i.e. 100 families & 30 & 60 & 120 \\
2:2, i.e. 200 families & 15 & 30 & 60 \\
1:2, i.e. 200 families & 15 & 30 & 60 \\
10:10, i.e. 1000 families & 3 & 6 & 12 \\
\hline
\end{tabular}


Effects of the QTL alleles were sampled from a gamma distribution with a shape parameter of 0.4 and a scale parameter of 1.66 [11]. There were no pleiotropic QTL effects, and no genetic or environmental correlation between the two traits. QTL effects were assumed to be either positive or negative with a probability of 0.5 , because the gamma distribution only gives positive values. After sampling, these QTL allelic effects were standardized such that the total genetic variance was equal to 1 for each trait, by calculating the standard deviation of the simulated genetic values of individuals from the last generation of the historical population, and scaling all QTL allelic effects by this standard deviation such that the genetic variance became 1 .

\section{Calculation of phenotypic values and true and estimated traditional BLUP and genome-wide breeding values}

The true genome-wide breeding values of an individual for CAND-TRAIT and SIB-TRAIT were calculated as:

$$
T B V_{i(\text { trait })}=\sum_{j=1}^{500} x_{i j} g_{j(\text { trait })},
$$

where $x_{i j}$ is the number of copies of allele 1 that individual $i$ has at the $j$ th QTL position and $g_{j(\text { trait })}$ is the allele substitution effect of allele 1 at the $j$ th QTL position for each trait. The phenotypic value of an individual for trait $t$ was simulated by adding an error term sampled from a normal distribution to the true breeding value $\left(T B V_{i(\text { trait })}\right)$ :

$$
y_{i(\text { trait })}=T B V_{i(\text { trait })}+\varepsilon_{i(\text { trait })},
$$

where $\varepsilon_{i \text { (trait) }}$ is an error term for individual $i$, which was normally distributed $N\left(0, \sigma_{e(t r a i t)}^{2}\right)$, and $\sigma_{e}^{2}$ was adjusted so that the heritability was 0.25 for CAND-TRAIT and 0.25 or 0.10 for SIB-TRAIT, which is common for such traits.

For TRAD-EBV, BLUP breeding values were calculated as in [2] by combining own performance and information of all available relatives from the current and earlier generations. The following statistical model used was:

$$
y_{i(\text { trait })}=\mu_{\text {trait }}+u_{i(\text { trait })}+e_{i(\text { trait })},
$$

where $y_{i(\text { trait })}$ is the record of individual $i ; \mu_{\text {trait }}$ is the overall mean, i.e. the only fixed effect in the model, $u_{i \text { (trait) }}$, is the additive genetic effect of individual $i$, which was correlated across individuals following the pedigreebased relationship matrix $\mathbf{A}$, and $e_{i \text { (trait })}$ is the error effect of individual $i$.

For $G W-E B V$, marker effects were predicted using the BLUP method as described in [3]. The statistical model used to estimate the marker effects for CAND-TRAIT and SIB-TRAIT followed method 2 of [12] and was:

$$
y_{i(\text { trait })}=\mu_{\text {trait }}+\sum_{j}^{n} X_{i j} a_{j(\text { trait })}+e_{i(\text { trait })},
$$

where $y_{i(\text { trait })}$ is the record of individual $i$ for CANDTRAIT and SIB-TRAIT; $X_{i j}=\frac{\left(Z_{i j}-2 p_{j}\right)}{\sqrt{2 p_{j}\left(1-p_{j}\right)}}$ denotes a standardized marker genotype (with mean 0 and variance 1$)$, where $Z_{i j}$ is the original marker genotype ( 0,1 or 2 alleles) and $p_{j}$ is the allele frequency at locus $j ; a_{j(\text { trait }}$ is the random effect of the $j t h$ marker, with $\operatorname{var}\left(a_{j(\text { trait })}\right)$ assumed to be $1 / n$, where $n$ is the number of markers (since the total genetic variance was standardised to 1 ); and $e_{i(\text { trait })}$ is a random residual.

Genome-wide breeding values were estimated by summing the estimates of the marker effects $\hat{a}_{j(\text { trait }) \text { : }}$

$$
E B V_{i(t r a i t)}=\sum_{j}^{n} X_{i j} \hat{a}_{j(t r a i t)} .
$$

In this model, all available information is used to estimate SNP effects, which implies that, as above, own performance and information on all available relatives from the current and earlier generations were used.

The relative economic weights for CAND-TRAIT and SIB-TRAIT in the total merit index used for selection were equal to $50 \%$, i.e.:

$$
E B V_{i(\text { index })}=0.5 E B V_{i(C A N D-T R A I T)}+0.5 E B V_{i(\text { SIB-TRAIT })} \text {. }
$$

\section{Statistics}

Summary statistics for each of the schemes were based on 100 replicated simulations. Selection schemes were run for ten generations (Generation0-Generation10). The breeding schemes were compared based on rates of inbreeding $(\Delta F)$ in Generation10, genetic gain for CAND-TRAIT and SIB-TRAIT (G, measured as genetic change in genetic standard deviation units $\left(\sigma_{a}\right)$ from generation Generation0 to generation Generation10), the percentage of total genetic gain that came from the SIB-TRAIT (\% SIB-TRAIT), and accuracy of the total merit index of the selection candidates $(A c c)$. Inbreeding coefficients $(F)$ were calculated based on pedigree information, assuming that individuals in Generation0 were unrelated base parents. Rates of inbreeding were calculated per generation. Accuracy of the total merit index of the selection candidates $(A c c)$ was calculated as the correlation between the true and estimated breeding values.

\section{Results}

Results from schemes with different numbers of selection candidates are in Table 2. For selection based on traditional BLUP estimated breeding values (TRAD$E B V$ ), factorial mating (e.g. changing the mating ratio 
Table 2 Effect of sire:dam mating ratio on genetic gains, rates of inbreeding and accuracy of selection with varying numbers of candidates

\begin{tabular}{|c|c|c|c|c|c|}
\hline $\begin{array}{l}\text { Sire:dam } \\
\text { mating ratio }\end{array}$ & $G_{\text {CAND-TRAIT }}{ }^{\mathrm{a}}\left(\sigma_{\mathrm{g}}\right)$ & $G_{S I B-T R A I T}{ }^{a}\left(\sigma_{g}\right)$ & $\begin{array}{l}\% \text { SIB- } \\
\text { TRAIT }\end{array}$ & $\Delta F^{\mathrm{b}}$ & $A c c^{c}$ \\
\hline \multicolumn{6}{|l|}{ Ncand $=3000$} \\
\hline \multicolumn{6}{|l|}{ TRAD-EBV } \\
\hline $1: 1$ & 0.36 & 0.17 & 32 & 0.014 & 0.492 \\
\hline $2: 2$ & 0.35 & 0.19 & 35 & 0.014 & 0.503 \\
\hline $1: 2$ & 0.34 & 0.16 & 32 & 0.011 & 0.489 \\
\hline $10: 10$ & 0.34 & 0.24 & 41 & 0.011 & 0.510 \\
\hline \multicolumn{6}{|l|}{ GW-EBV } \\
\hline $1: 1$ & 0.37 & 0.35 & 49 & 0.010 & 0.728 \\
\hline $2: 2$ & 0.36 & 0.36 & 50 & 0.010 & 0.718 \\
\hline $1: 2$ & 0.35 & 0.33 & 48 & 0.011 & 0.716 \\
\hline $10: 10$ & 0.38 & 0.35 & 48 & 0.011 & 0.711 \\
\hline \multicolumn{6}{|l|}{ Ncand $=6000$} \\
\hline \multicolumn{6}{|l|}{ TRAD-EBV } \\
\hline $1: 1$ & 0.39 & 0.16 & 29 & 0.015 & 0.501 \\
\hline $2: 2$ & 0.38 & 0.20 & 34 & 0.015 & 0.504 \\
\hline $1: 2$ & 0.38 & 0.16 & 30 & 0.011 & 0.506 \\
\hline $10: 10$ & 0.39 & 0.23 & 37 & 0.013 & 0.514 \\
\hline \multicolumn{6}{|l|}{ GW-EBV } \\
\hline $1: 1$ & 0.42 & 0.37 & 47 & 0.008 & 0.754 \\
\hline $2: 2$ & 0.42 & 0.37 & 47 & 0.007 & 0.743 \\
\hline $1: 2$ & 0.41 & 0.35 & 46 & 0.005 & 0.738 \\
\hline $10: 10$ & 0.43 & 0.37 & 46 & 0.005 & 0.734 \\
\hline \multicolumn{6}{|c|}{ Ncand $=12,000$} \\
\hline \multicolumn{6}{|l|}{ TRAD-EBV } \\
\hline $1: 1$ & 0.43 & 0.13 & 24 & 0.015 & 0.490 \\
\hline $2: 2$ & 0.42 & 0.19 & 31 & 0.014 & 0.514 \\
\hline $1: 2$ & 0.42 & 0.15 & 26 & 0.011 & 0.501 \\
\hline $10: 10$ & 0.43 & 0.23 & 35 & 0.014 & 0.518 \\
\hline \multicolumn{6}{|l|}{ GW-EBV } \\
\hline 1:1 & 0.44 & 0.39 & 47 & 0.009 & 0.767 \\
\hline $2: 2$ & 0.45 & 0.40 & 47 & 0.008 & 0.761 \\
\hline $1: 2$ & 0.44 & 0.40 & 47 & 0.007 & 0.754 \\
\hline $10: 10$ & 0.46 & 0.40 & 46 & 0.006 & 0.753 \\
\hline
\end{tabular}

Genetic gain (G) for CAND-TRAIT and SIB-TRAIT, percentage of total genetic gain that comes from the SIB-TRAIT (\% SIBTRAIT), rates of inbreeding $(\triangle F)$ and accuracy of the total merit index of the selection candidates (Acc) in Generation 10 for schemes with different number of candidates; 100 sires and 100 dams were selected for schemes with mating ratio $1: 1,2: 2$ and 10:10 and 100 sires and 200 dams for schemes with mating ratio 1:2; heritability of $S I B$ TRAIT $=0.25 ;$ Ntest $=3000$

a Standard errors between 0.0002 and 0.0006

b Standard errors between 0.000000 and 0.000006

c Standard errors between 0.0001 and 0.0004

from 1:1 to 10:10 with a constant number of parents) substantially increased genetic gain for the SIB-TRAIT (from 0.17 to $0.24 \sigma_{g}, 0.16$ to $0.23 \sigma_{g}$, and 0.13 to $0.23 \sigma_{g}$ for Ncand = 3000, 6000 and 12,000, respectively), while genetic gain for CAND-TRAIT was unchanged or only slightly reduced. Consequently, total genetic gain was greater for factorial mating designs, as was the \% SIBTRAIT. Genetic gains with mating ratios 1:2 and 2:2 were, as expected, intermediate between those obtained with the two most extreme designs; with mating ratio $2: 2$, the genetic gain for SIB-TRAIT was slightly larger than with mating ratio 1:2. Despite faster genetic gain, the 10:10 mating ratio had lower rates of inbreeding than the $1: 1$ mating ratio (from 0.014 to $0.011,0.015$ to 0.013 , and 0.008 to 0.005 for Ncand $=3000,6000$ and 12,000, respectively). Acc tended to increase when the mating ratio changed from 1:1 to $10: 10$.

Selection on genome-wide breeding values $(G W-E B V)$ achieved higher genetic gains than selection on traditional breeding values (TRAD-EBV), especially for $S I B$ TRAIT. However, the most striking result of the schemes based on $G W-E B V$ was that factorial mating generally had a limited effect on genetic gain for both traits $(+0$ to $3 \%$ for CAND-TRAIT; +1 to $5 \%$ for SIB-TRAIT), and thus also on the \% SIB-TRAIT. Rates of inbreeding were considerably lower for all genome-wide selection schemes than for traditional selection. Furthermore, when changing the mating ratio from $1: 1$ to $10: 10$, rates of inbreeding decreased from 0.015 to 0.014 and from 0.009 to 0.006 for $N$ cand $=6000$ and 12,000, respectively, but not with Ncand $=3000$. Acc tended to decrease when the mating ratio increased from 1:1 to 10:10.

The results for the scheme with a lower heritability (0.10) for SIB-TRAIT (Ncand $=6000$ ) are in Table 3 . As expected, the lower heritability resulted in a shift of genetic gain towards CAND-TRAIT (giving somewhat increased genetic gain for this trait), while genetic gain for SIB-TRAIT was substantially reduced. With TRAD$E B V$, factorial mating resulted in considerably greater genetic gain for SIB-TRAIT, with increases that were of similar magnitude as for the scheme with a heritability of 0.25 for SIB-TRAIT, but had little effect on genetic gain for CAND-TRAIT. Again, rates of inbreeding were lower for the factorial mating designs $(0.005$ for $10: 10$ and 0.008 for 1:1). Also with $G W-E B V$, factorial mating had little effect on genetic gain, but a substantial effect on rates of inbreeding.

Results for the scenario with fewer individuals in the sib test (Ntest) having phenotypic records on SIB-TRAIT are in Table 4. Reducing the number of sibs with phenotypic records had a similar effect as reducing the heritability of SIB-TRAIT (although the effects were less strong), since in both scenarios the amount of information on sibs is reduced, either because the information content of each sib phenotype for the SIB-TRAIT is reduced (lower heritability) or because the number of phenotypes recorded on sibs is reduced. 
Table 3 Effect of sire:dam mating ratio on genetic gains, rates of inbreeding and accuracy of selection with a lower heritability for SIB-TRAIT

\begin{tabular}{llllll}
\hline $\begin{array}{l}\text { Sire:dam } \\
\text { mating ratio }\end{array}$ & $G_{\text {CAND-TRAIT }}{ }^{\mathbf{a}}\left(\sigma_{\mathbf{g}}\right)$ & $G_{\text {SIB-TRAIT }}{ }^{\mathbf{a}}\left(\sigma_{\mathbf{g}}\right)$ & $\begin{array}{l}\% \text { SIB- } \\
\text { TRAIT }\end{array}$ & $\Delta F^{\mathbf{b}}$ & ACC $^{\mathbf{c}}$ \\
\hline TRAD-EBV & & & & & \\
$1: 1$ & 0.44 & 0.05 & 10 & 0.015 & 0.525 \\
$2: 2$ & 0.45 & 0.07 & 13 & 0.014 & 0.526 \\
$1: 2$ & 0.43 & 0.04 & 9 & 0.010 & 0.518 \\
$10: 10$ & 0.45 & 0.07 & 13 & 0.011 & 0.514 \\
GW-EBV & & & & & \\
$1: 1$ & 0.51 & 0.14 & 22 & 0.008 & 0.744 \\
$2: 2$ & 0.51 & 0.15 & 23 & 0.007 & 0.731 \\
$1: 2$ & 0.50 & 0.15 & 23 & 0.005 & 0.728 \\
$10: 10$ & 0.51 & 0.15 & 23 & 0.005 & 0.715 \\
\hline
\end{tabular}

Genetic gain (G) for CAND-TRAIT and SIB-TRAIT, percentage of total genetic gain that comes from the SIB-TRAIT (\% SIB-TRAIT), rates of inbreeding $(\triangle F)$ and accuracy of the total merit index of the selection candidates (Acc) in Generation 10 for schemes with lower heritability of SIB-TRAIT of $0.10 ; 100$ sires and 100 dams were selected for schemes with mating ratios 1:1, 2:2 and 10:10 and 100 sires and 200 dams for schemes with mating ratio 1:2; Ntest $=3000$; Ncand $=6000$

a Standard errors between 0.0002 and 0.0006

b Standard errors between 0.000000 and 0.000003

c Standard errors between 0.0001 and 0.0002

Table 4 Effect of sire:dam mating ratio on genetic gains, rates of inbreeding and accuracy of selection with fewer tested sibs

\begin{tabular}{llllll}
\hline $\begin{array}{l}\text { Sire:dam } \\
\text { mating ratio }\end{array}$ & $\boldsymbol{G}_{\text {CAND-TRAIT }}{ }^{\mathbf{a}}\left(\sigma_{\mathbf{g}}\right)$ & $\boldsymbol{G}_{\text {SIB-TRAIT }}{ }^{\mathbf{a}}\left(\sigma_{\mathbf{g}}\right)$ & $\begin{array}{c}\text { \% SIB- } \\
\text { TRAIT }\end{array}$ & $\Delta \boldsymbol{F}^{\mathbf{b}}$ & ACC $^{\mathbf{c}}$ \\
\hline TRAD-EBV & & & & & \\
$1: 1$ & 0.41 & 0.11 & 21 & 0.015 & 0.461 \\
$2: 2$ & 0.40 & 0.14 & 26 & 0.015 & 0.471 \\
$1: 2$ & 0.40 & 0.11 & 22 & 0.011 & 0.463 \\
$10: 10$ & 0.41 & 0.17 & 29 & 0.11 & 0.472 \\
GW-EBV & & & & & \\
$1: 1$ & 0.44 & 0.31 & 41 & 0.008 & 0.694 \\
$2: 2$ & 0.44 & 0.32 & 42 & 0.007 & 0.689 \\
$1: 2$ & 0.44 & 0.30 & 41 & 0.006 & 0.682 \\
$10: 10$ & 0.44 & 0.32 & 42 & 0.005 & 0.672 \\
\hline
\end{tabular}

Genetic gain (G) for CAND-TRAIT and SIB-TRAIT, percentage of total genetic gain that comes from the SIB-TRAIT (\% SIB-TRAIT), rates of inbreeding $(\triangle F)$, and accuracy of the total merit index of the selection candidates (Acc) in Generation 10 for schemes with a sib test using fewer individuals, Ntest $=1000$; 100 sires and 100 dams were selected for schemes with mating ratios 1:1, 2:2 and 10:10 and 100 sires and 200 dams for schemes with mating ratio 1:2; Heritability for SIB-TRAIT $=0.25 ;$ Ncand $=6000$

a Standard errors between 0.0002 and 0.0006

b Standard errors between 0.000000 and 0.000006

c Standard errors between 0.0001 and 0.0004

\section{Discussion}

The main result of this study is that with genome-wide estimated breeding values, the effect of sire:dam mating ratio on genetic gain was less than $5 \%$ for both traits when selecting simultaneously for CAND-TRAIT and SIB-TRAIT. The offspring (total number ranging from 6000 to 15,000 ) were split over the 100,200 or 1000 families, i.e. each individual had many sibs, either full-sibs or half-sibs, with the different mating ratios. In the schemes where both CAND-TRAIT and SIB-TRAIT had a heritability of 0.25 and both Ntest and Ncand were equal to 3000 (Table 2), genetic gains were similar for SIB-TRAIT and CAND-TRAIT, although only CAND-TRAIT was measured on the selection candidates. Having genomic information on both candidates and their sibs enabled within-family selection for both traits, which explains the higher genetic gain obtained for schemes based on genome-wide breeding values.

Several other studies on aquaculture species have shown that factorial mating designs were beneficial to maintain low rates of inbreeding and increase genetic gains (e.g. $[13,14])$ using phenotypic selection or selection on traditional BLUP estimated breeding values and genome-wide estimated breeding values [15]. We confirmed those results, and showed that rates of inbreeding decreased by $\sim 20$ to $30 \%$ for both traditional and genomic selection schemes when changing the sire:dam mating ratio from 1:1 to 10:10. For factorial mating systems with more mates per sire and/or dam, more (but smaller) families are produced from the same number of parents. Indeed, for the schemes in Table 2 with Ncand $=6000$, the number of full-sib families from which parents were selected increased from 45 to 141 for schemes based on genome-wide estimated breeding values and from 24 to 84 for schemes based on traditional BLUP estimated breeding values, when the mating ratio changed from 1:1 to 10:10. Thus, in schemes with a 10:10 mating ratio, the best parents are mated with many partners, thus increasing the probability of combining favorable sires and dams, whereas in schemes with a 1:1 and to some extent a $2: 2$ mating ratio, the best parents can by chance mate with inferior partners. Hence, the number of superior families decreases as the number of matings per parent decreases. This explains that, in schemes based on traditional BLUP estimated breeding values, genetic gain for SIB-TRAIT was smaller for a mating ratio of 1:1 than for a mating ratio of 10:10 (since selection was only based on family means). It also explains the higher rates of inbreeding for the mating ratio 1:1 compared to 10:10, since parents are to a larger extent selected within the fewer superior families (using either random or genomic within-family selection).

Mating ratio had a small effect on the accuracy of estimated breeding values of schemes with either traditional or genome-wide breeding values when changing the mating ratio by keeping the number of parents constant 
but mating them to different numbers of mates. For the scheme in Table 2 with Ncand $=6000$, Acc of the TRAD$E B V$ was equal to 0.501 and 0.514 for mating ratios $1: 1$ and 10:10, respectively. Hence, the increase in genetic gain with more mates per sire and/or dam in the factorial mating design was mainly due to greater selection intensity rather than accuracy, i.e. when changing the mating ratio from 1:1 to $10: 10$ results in 1000 instead of 100 families to select from. Thus, our findings confirmed the results of $[1,16]$, which showed that increasing the number of mates mainly affects selection intensity. $A c c$ of the $G W-E B V$ was equal to 0.754 and 0.734 for mating ratios $1: 1$ and $10: 10$, respectively. This decrease in $A c c$ can be explained by a decrease in genetic variance during selection in earlier generations, which was not observed in Generation2 (result not shown). Thus, with the $G W-E B V$ schemes, increasing the number of mates per sire and/or dam in the factorial mating design led to little change in accuracy, selection intensity and genetic gain because family information is relatively less important due to the greater within-family component compared to selection on TRAD-EBV.

Overall, selection on $G W-E B V$ increased genetic gain for CAND-TRAIT by 4 to $15 \%$ and for SIB_TRAIT by 50 to $240 \%$, compared with selection on TRAD-EBV. When the heritabilities of CAND-TRAIT and SIB-TRAIT were identical, selection on $G W-E B V$ led to similar genetic gains for both traits, although SIB-TRAIT was measured on sibs of the candidates only. However, with a smaller number of test individuals or a larger genome (i.e. with more chromosomes, genomic relationships between sibs become closer to their expectation of 0.5 , and differences in relationships are smaller, resulting in less accurate genomic selection), genetic gain for the SIB-TRAIT would likely have been somewhat smaller.

In this study, comparisons between breeding schemes was done without a restriction on rate of inbreeding and showed that rates of inbreeding were $\sim 50 \%$ lower with selection on GW-EBV than on TRAD-EBV. Thus, if restrictions are imposed on rate of inbreeding rather than on the number of parents and offspring per parent, genetic gains are expected to be higher than observed here and to be higher for the schemes based on GW-EBV than on TRAD-EBV. However, the rates of inbreeding presented here were based on pedigree-based relationships, which were shown by [16] to underestimate genome-based inbreeding for schemes with selection on GW-EBV. The lower rates of inbreeding found by increasing the number of mates per sire and/or dam in the factorial mating designs should allow, in practice, for more intense selection on both traits for both traditional and genomic selection, and thus higher genetic gain.

As a test, $G W-E B V$ schemes were also run with a fixed tank effect in the model (results not shown). Overall, similar results were obtained for all mating ratios, but all genetic gains were slightly reduced, because the number of degrees of freedom of the model was increased since more effects were fitted, and thus the accuracy of the breeding values decreased.

In this study, CAND-TRAIT and SIB-TRAIT were assumed to be uncorrelated. If the genetic correlation differs from 0, the SIB-TRAIT can be separated into a component that can be predicted by CAND-TRAIT records (which was recorded on the candidates) and a component that is uncorrelated to CAND-TRAIT and thus cannot be predicted from CAND-TRAIT records. The latter component would thus be similar to the uncorrelated SIB-TRAIT considered in this study, and the combination of CAND-TRAIT and the predictable component of SIB-TRAIT would be like the CAND-TRAIT in our study. This decomposition of the SIB-TRAIT may alter the relative importance of the two traits, but the effect of an altered importance of the SIB-TRAIT was found to be small in the current study (Table 3). Hence, our general results are not expected to be sensitive to a non-zero correlation between the CAND-TRAIT and SIB-TRAIT.

For all mating ratios, we used the same number of parents and offspring (selection candidates and test-sibs), except for mating ratio 1:2. Thus, the same number of individuals was phenotyped and genotyped and, any differences in cost are due to differences in the number of families used. For practical family-based breeding schemes, the number of families is the largest limitation, because of the high investment and running costs for each family tank, which are required to raise families until tagging size. At least 50 single-pair mated families are needed to maintain inbreeding rate within the generally recommended value of $1 \%$ [17]. For genomic selection, separate family tanks may not be needed, because the relationships among individuals can be established based on data from genetic markers. Thus, a larger number of families and a larger total number of animals can be realized more easily, such that increased selection intensity is possible at the same rate of inbreeding. Without family tanks, it will be necessary to estimate marker effects in a separate population of sibs that are raised in addition to the candidates. Management of these two populations must be optimized such that the family contributions are similar.

\section{Conclusions}

Changing the sire:dam mating ratio from 1:1 to $10: 10$ increased genetic gain substantially with $T R A D-E B V$, mainly through increased selection intensity for the $S I B$ $T R A I T$, whereas with $G W-E B V$, it had a limited effect on genetic gain for both traits. Rates of inbreeding decreased for both selection methods. 


\section{Authors' contributions}

AKS performed computer simulations, and drafted the manuscript. JØ participated in the decisions for the simulated mating designs, and contributed in writing the manuscript. All authors read and approved the final manuscript.

\section{Author details}

${ }^{1}$ Nofima AS, P.O. Box 5010, 1432 Ås, Norway. ${ }^{2}$ AquaGen AS, P.O. Box 1240 , Sluppen, 7462 Trondheim, Norway.

\section{Acknowledgements}

This study was supported by Grant 190442 from the Research Council of Norway. Calculations were done on the ABEL computer cluster at University of Oslo, Norway.

\section{Competing interests}

The authors declare that they have no competing interests.

Received: 22 July 2015 Accepted: 11 June 2016

Published online: 24 June 2016

\section{References}

1. Woolliams JA. Modifications to MOET nucleus breeding schemes to improve rates of genetic progress and decrease rates of inbreeding in dairy cattle. Anim Prod. 1989:49:1-14.

2. Henderson C. Applications of linear models in animal breeding. Guelph: Guelph University Press; 1984.

3. Meuwissen THE, Hayes BJ, Goddard ME. Prediction of total genetic value using genome-wide dense marker maps. Genetics. 2001;157:1819-29.

4. Daetwyler HD, Villanueva B, Bijma P, Woolliams JA. Inbreeding in genomewide selection. J Anim Breed Genet. 2007;124:369-76.
5. Fisher R. The genetical theory of natural selection. Oxford: Clarendon Press; 1930.

6. Wright S. Evolution in Mendelian populations. Genet Sel Evol. 1931;16:97-159.

7. Sonesson AK, Meuwissen THE. Testing strategies for genomic selection in aquaculture breeding programs. Genet Sel Evol. 2009;41:37.

8. Kimura M. The number of heterozygous nucleotide sites maintained in a finite population due to steady flux of mutations. Genetics. 1969:61:893-903.

9. Roach JC, Glusman G, Smit AFA, Huff CD, Hubley R, Shannon PT, et al. Analysis of genetic inheritance in a family quartet by whole-genome sequencing. Science. 2010;328:636-9.

10. Haldane JBS. The combination of linkage values, and the calculation of distances between the loci of linked factors. J Genet. 1919;8:299-309.

11. Hayes B, Goddard ME. The distribution of the effects of genes affecting quantitative traits in livestock. Genet Sel Evol. 2001:33:209-29.

12. VanRaden PM. Efficient methods to compute genomic predictions. J Dairy Sci. 2008:91:4414-23.

13. Dupont-Nivet M, Vandeputte M, Chevassus B. Optimization of factorial mating designs for inference on heritability in fish species. Aquaculture. 2002;204:361-70

14. Busack C, Knudsen CM. Using factorial mating designs to increase the effective number of breeders in fish hatcheries. Aquaculture. 2007;273:24-32.

15. Nirea KG, Sonesson AK, Woolliams JA, Meuwissen THE. Effect of nonrandom mating on genomic and BLUP selection schemes. Genet Sel Evol. 2012:44:11.

16. Sonesson AK, Woolliams JA, Meuwissen THE. Genomic selection requires genomic control of inbreeding. Genet Sel Evol. 2012:44:27.

17. Bentsen $\mathrm{HB}$, Olesen I. Designing aquaculture mass selection programs to avoid high inbreeding rates. Aquaculture. 2002;204:349-59.

\section{Submit your next manuscript to BioMed Central and we will help you at every step:}

- We accept pre-submission inquiries

- Our selector tool helps you to find the most relevant journal

- We provide round the clock customer support

- Convenient online submission

- Thorough peer review

- Inclusion in PubMed and all major indexing services

- Maximum visibility for your research

Submit your manuscript at www.biomedcentral.com/submit 